\title{
282. Vor- und Nachteile der äußeren Stabilisation bei Beckenfrakturen
}

\author{
H.-J. Egbers, D. Havemann und L. Schroeder \\ Abteilung Unfallchirurgie im Klinikum der Christian-Albrechts-Universität Kiel, \\ Hospitalstraße 40, D-2300 Kiel 1
}

\section{The Advantages and Disadvantages of External Fixation in Pelvic Fractures}

\begin{abstract}
Summary. The indication for the use of external fixation in pelvic fractures is determined by the seriousness of the injury and the instability of the pelvic girdle. The advantages of improved techniques in intensive care and early functional therapy, as well as minimized traumatization, reduction of thrombo-embolic phenomena and diminution of retroperitoneal blood loss, far outweigh the disadvantages, namely risk of infection and possible loss of stability. Computer tomography and experimental examinations for strength determination are also discussed.
\end{abstract}

Key words: External fixation - Pelvic fractures - Advantages - Disadvantages.

Zusammenfassung. Die Indikation zur Anwendung des Fixateur externe bei Beckenringverletzungen wird bestimmt durch den Gesamtverletzungsschweregrad und die Instabilität des Beckenringes. Die Vorteile mit verbesserten Möglichkeiten der Intensivpflege und frühfunktioneller Behandlung sowie geringer Traumatisierung, Verringerung des Thrombose- und Embolierisikos und Minderung des retroperitonealen Blutverlustes überwiegen gegenüber den Nachteilen mit Infektionsrisiko und möglichem Stabilitätsverlust. Computertomographie und experimentelle Untersuchungen zur Festigkeitsbeurteilung werden besprochen.

Schliisselwörter: Äußere Stabilisation - Beckenfrakturen - Vor- und Nachteile.

\section{Plattenfreie Synthese der ligamentären Beckenringverletzung (Malgaigne-Luxation)}

\author{
R. Labitzke und U. Witzel
}

Abteilung für Chirurgie und Unfallchirurgie, Evangelisches Krankenhaus GmbH, D-5840 Schwerte/R.

\section{“Sleeve-Cable Tensioning” of Ligamentary Pelvic Luxation (Malgaigne)}

Summary. The harmony of motion within the pelvic girdle depends on the undisturbed and freely co-ordinated motility of the iliosacral joints and the symphysis. Plate osteosynthesis interrupts this system which is very important to the overall picture. "Sleeve-cable tensioning" enables dynamic fixation of the re-united pelvic girdle. Cables - guided through intra-osseous pressure-distributing sleeves - brace the symphysis as well as both dorsal iliac spines. The biomechanical advantages of the described technique could make it the treatment of choice for injuries like Malgaigne luxation.

Key words: Sleeve-cable tensioning - Malgaigne luxation.

Zusammenfassung. Die Bewegungsharmonie zwischen lumbosacralem Übergang und Becken wird durch eine belastungskonforme, subtil abgestufte Motilität innerhalb des elastischen Beckenringes durch funktionstüchtige Ileosacralgelenke und die Symphyse aufrecht erhalten. Daher kann nur ein elastisches Verfahren zur Beseitigung einer Beckenringluxation biomechanisch sinnvoll sein. Versteifende Synthesen hingegen setzen dieses auf interne Relativbewegungen begründete System matt und zeigen in über $50 \%$ unbefriedigende Ergebnisse. Die „Hülsen-Seil-Verspannung“ erlaubt - als einzige Methode überhaupt - eine dynamische Fixation des Beckenringes, die die physiologischen Reize zur Heilung der gerissenen Kapselbandapparate erhält. Die Verspannung mit hochbelastbaren Drahtseilen (Labitzke 1979) geschieht über intraossäre Druckverteilungshülsen zwischen den dorsalen Darmbeinstacheln einerseits und den beiden Schambeinästen andererseits. Biomechanisch ist die operativ einfach durchzuführende Methode so überzeugend, daß sie zur Therapie der Wahl für ligamentäre Verletzungen werden könnte.

Schliisselwörter: Hülsen-Seil-Verspannung - Malgaigne Luxation. 